[0212-7199 (2005) 22: 2; pp 65-68] ANALES DE MEDICINA INTERNA Copyright (C) 2005 ARAN EDICIONES, S.L.

AN. MED. INTERNA (Madrid) Vol. $22, \mathrm{~N}^{\circ} 2$, pp. 65-68, 2005

\title{
Producción española en revistas internacionales en el área de técnicas diagnósticas y terapéuticas del sistema respiratorio en el periodo 1990 a 2002
}

\author{
J. I. DE GRANDA ORIVE, F. GARCÍA RÍO'ㄹ, V. GALLEGO RODRÍGUEZ², \\ J. A. ESCOBAR SACRISTÁN ${ }^{2}$, J. JAREÑO ESTEBAN ${ }^{2}$, L. CALLOL SÁNCHEZ ${ }^{2}$
}

Servicios de Neumología. Hospital General Básico de la Defensa. Valencia. ${ }^{\text {Hospital }}$ Universitario La Paz. ${ }^{2}$ Hospital Central de la Defensa. Madrid

SPANISH SCIENTIFIC PRODUCTION IN DIAGNOSTIC AND THERAPEUTIC RESEARCH AREA OF RESPIRATORY SYSTEM IN INTERNATIONAL JOURNALS FROM 1990 TO 2002

\section{RESUMEN}

Objetivo: El objetivo de este trabajo fue determinar la aportación de autores españoles a la ciencia internacional en el área temática de técnicas diagnósticas y terapéuticas del sistema respiratorio, en el período 1990 a 2002.

Material y métodos: Se efectuó una búsqueda de los artículos publicados entre 1990 a 2002 incluidos en PUB-MED. La estrategia de busca se centró en las palabras clave (PC): respiratory system y en aquellas relacionadas con los procedimientos diagnósticos y terapéuticos empleados en sistema respiratorio.

Resultados: Se obtuvieron 67 artículos, sobre el motivo a estudio, de autores españoles publicados en revistas internacionales. Se ha mantenido una producción estable a lo largo de los años entre 2 y 5 artículos por año, siendo los neumólogos los más productivos en este tema. Aunque existe una dispersión de la producción según la distribución de los artículos por provincias e instituciones españolas fue Barcelona $(43,1 \%)$ y el Hospital Clinic i Provincial de Barcelona $(23,8 \%)$ quienes concentraron un mayor número de artículos en el período estudiado. Los procedimientos diagnóstico terapéuticos más productivos fueron el lavado broncoalveolar y el uso del catéter telescopado en la fibrobroncoscopia.

Conclusiones: La producción, en el área de técnicas diagnósticas y terapéuticas de sistema respiratorio en revistas internacionales, se mantiene estable, sin una tendencia definida, lo que contrasta con el gran aumento de producción que ha experimentado el sistema respiratorio.

PALABRAS CLAVE: Información científica. Bibliometría. Respiratorio. Broncoscopia.

\section{ABSTRACT}

Objective: The aim of this study were: to analyze the spanish production in diagnostic and therapeutics research area of respiratory sys tem in international journals from 1990 to 2002.

Material and methods: Papers published in diagnostic and therapeutics area of respiratory system during this period of time were selected by the PUB-MED system. We delimited the production with key words: respiratory system and the one that is used by the diagnostic and therapeutics research area.

Results: We obtain a total of 67 document published in international journals by spanish authors. The scientific production in the diagnostic and therapeutics area have stabilized in this 12-years period. The distribution of articles by the institutional affiliation and province of authors also showed a wide dispersion: Barcelona and Clinic and Provincial Hospital of Barcelona were responsible for 43,1\% and 23,8\% of all the production. Bronchoalveolar lavage and the use of telescopic catheter were the type of diagnostic and therapeutic procedure more productive.

Conclusions: The scientific production in the diagnostic and therapeutics area of respiratory system have stabilized in this 12-years period, this evidence contrast with the high growth detected in respiratory system in general.

KEY WORDS: Scientific information. Bibliometry. Respiratory. Broncoscopy.

de Granda Orive JI, García Río F, Gallego Rodríguez, V, Escobar Sacristán JA, Jareño Esteban J, Callol Sánchez L. Producción española en revistas internacionales en el área de técnicas diagnósticas y terapéuticas del sistema respiratorio en el periodo 1990 a 2002. An Med Interna (Madrid) 2005; 22: 65-68

\section{INTRODUCCIÓN}

En la medicina moderna existe una importancia creciente de las áreas de técnicas diagnósticas y terapéuticas (TDT) especializadas, lo cual no es diferente en el sistema respiratorio. En esta área de la clínica, se han ido incorporando a lo largo de los últimos años una serie de técnicas, basadas en parte en la tecnología y, que han mejorado la tasa de diagnósticos específicos.
Las palabras clave o descriptores suponen una herramienta imprescindible a la hora de realizar una búsqueda bibliográfica. Permiten el acceso, en las grandes bases de datos, a todos los trabajos relacionados (1). Los estudios bibliométricos estudian el tamaño, el crecimiento y la distribución de los documentos científicos. El objetivo de este trabajo fue determinar la aportación de autores españoles a la ciencia internacional en el área temática de técnicas diagnósticas y terapéuticas del sistema respiratorio, en el periodo 1990 a 2002. 


\section{MATERIAL Y MÉTODOS}

- Recogida de datos: Se efectúo una búsqueda de los artículos publicados entre 1990 y 2002 incluidos en PUB-MED (2). La estrategia de búsqueda se centró en las palabras clave (PC): respiratory system y en aquellas relacionadas con el término broncoscopia y procedimientos diagnósticos y terapéuticos empleados en el sistema respiratorio. Se delimitó la búsqueda mediante la palabra "Spain" en el epígrafe "Affiliation", y se recogieron todos aquellos documentos en los que la primera institución firmante resultó ser española.

Las PC empleadas (Tabla I) fueron definidas previamente mediante un proceso de revisión manual de todos los artículos originales publicados en la principal revista de habla hispana en sistema respiratorio [Archivos de Bronconeumología, (AB)] a lo largo del periodo 1994 a 2000 (3-5). Los descriptores hallados se tradujeron al inglés (http://decs.bvs.br) y se verificaron con los utilizados por el MeSH Browser del Index Medicus. La exactitud de las PC una vez traducidas fue comparada con las que la propia revista $\mathrm{AB}$ publica traducidas al inglés. Lo anterior se realizó en una muestra aleatoria de 25 originales observándose una traducción equiparable en el $100 \%$ de las palabras correctas elegidas.

\section{TABLA I}

PALABRAS CLAVE UTILIZADAS PARA LA BÚSOUEDA DE ARTÍCULOS DEL ÁREA DE TÉCNICAS DIAGNÓSTICAS Y TERAPÉUTICAS DE SISTEMA RESPIRATORIO EN REVISTAS INTERNACIONALES

\section{- Respiratory system}

-Bronchoscopy OR Bronchoscopes OR Endoscopy OR

Bronchoalveolar Fluid OR Biopsy, Needle OR Diagnostic Techniques OR Surgical Endoscopy OR Pulmonary surgical procedures OR Laser therapy low-level OR Laser surgery OR Brachiterapy

-Análisis estadístico: El análisis estadístico de los datos se realizó mediante el programa SPSS versión 8.0 para Windows (SPSS, Inc., Illinois. USA). El análisis estadístico fue descriptivo expresando las variables cuantitativas como la media aritmética ( \pm desviación estándar) de la variable. Para la comparación de proporciones se utilizó el test del Chi-cuadrado para variables cualitativas para datos independientes. Para la comparación de las medias de dos grupos para una variable cuantitativa continua se utilizó la prueba de la t de Student, tras comprobar el ajuste a la distribución normal mediante la prueba de Kolmogorov-Smirnoff. Se seleccionó como nivel de significación estadística un valor de la $\mathrm{p}$ inferior a 0,05 (intervalo de confianza del 95\%).

\section{RESULTADOS}

Fueron encontrados 176 artículos de autores españoles en revistas internacionales sobre el tópico a estudio. Se realizó una revisión manual de los artículos hallados, pues las palabras clave localizadas también son utilizadas en otras áreas temáticas de la ciencia médica, no haciendo distinciones el $\mathrm{MeSH}$ Browser entre los términos utilizados de forma general entre las distintas técnicas de las diferentes áreas clínicas. Se obtuvieron 67 artículos sobre TDT en sistema respiratorio en revistas internacionales en el periodo analizado.

La media del índice de cooperación $( \pm \mathrm{DE})$ fue de $5 \pm 2$ autores (intervalo de 1 a 9 autores). La distribución de los artículos por años analizados se ha mantenido estable en el tiempo entre 2 y 5 artículos por año salvo en 1998 y 1999 que tienen 12 y 10 artículos respectivamente. Los tipos de artículo más frecuentemente publicados son los originales con 51 documentos $(76,1 \%)$ y casos clínicos con 13 documentos $(19,4 \%)$, estando el $95,5 \%$ del total (64) publicados en inglés. La distribución por revistas la podemos observar en la tabla II. En cuanto a las especialidades más productivas, como no podía ser de otra manera, han sido neumología con el $61,1 \%$ (41) de los artículos y cirugía torácica con el 11,9\%. Aunque la distribución por centros o instituciones de trabajo está ampliamente representada por toda la geografía española (Tabla III), claramente el mayor foco de producción es el Hospital Clinic i Provincial de Barcelona con 16 artículos $(23,8 \%)$. En el total de artículos y en cuanto a la distribución por provincias españolas (Tabla III), Barcelona ha producido el 43,1\% de lo documentos y Madrid el 22,3\% de los mismos. En la tabla IV se encuentra la distribución de los documentos según el procedimiento terapéutico-diagnóstico utilizado. No hemos encontrado diferencias estadísticamente significativas

TABLA ॥

DISTRIBUCIÓN DE LOS ARTÍCULOS POR REVISTA DE PROCEDENCIA

\begin{tabular}{lc}
\hline Revista (List Journal Indexed) & Frecuencia \\
\hline Eur Respir J & 13 \\
AJRCCM/ARRD & 9 \\
Chest & 9 \\
Thorax & 5 \\
Eur J Cardiothorac Surg & 3 \\
Arch Bronconeumol & 2 \\
Monaldi Arch Chest Dis & 2 \\
Pediatr Surg Int & 2 \\
Respir Med & 2 \\
Respiration & 2 \\
Ann Oncol & 1 \\
Ann Thorac Surg & 1 \\
Bone Marrow Transplant & 1 \\
Clin Pharmacol Ther & 1 \\
Crit Care Med & 1 \\
Enferm Infec Microbiol Clin & 1 \\
Eur J Pediatr Surg & 1 \\
Eur J Surg & 1 \\
In vivo & 1 \\
Infect Dis Clin North Am & 1 \\
J Antimicrob Chemother & 1 \\
J Cardiovasc Surg & 1 \\
J Thorac Imaging & 1 \\
Postgrad Med & 1 \\
Surg Laparosc Endosc & 1 \\
Transplant Proc & 1 \\
Transplantation & 1 \\
Tumori & 1 \\
\hline Total & 1 \\
\hline & 1 \\
\hline
\end{tabular}


TABLA III

DISTRIBUCIÓN DE LOS ARTÍCULOS POR PROVINCIAS E INSTITUCIONES

\begin{tabular}{|c|c|}
\hline Institución & Frecuencia \\
\hline Hospital Clinic i Provincial & 16 \\
\hline Hospital Nuestra Señora del Pino & 6 \\
\hline Hospital Germans Trias y Pujol & 4 \\
\hline Hospital Universitario La Paz & 4 \\
\hline Hospital Reina Sofía & 3 \\
\hline Hospital Universitario 12 de Octubre & 3 \\
\hline Hospital Vall d'Hebron & 3 \\
\hline Hospital de Bellvitge & 2 \\
\hline Hospital de Cruces & 2 \\
\hline Hospital de La Princesa & 2 \\
\hline Hospital General de Alicante & 2 \\
\hline Hospital Gregorio Marañón & 2 \\
\hline Hospital La Fe & 2 \\
\hline Hospital Miguel Servet & 2 \\
\hline Hospital Ramón y Cajal & 2 \\
\hline Clínica de N y CT Villarroel & 1 \\
\hline Fundación Jiménez Díaz & 1 \\
\hline Hospital Arnau de Vilanova & 1 \\
\hline Hospital Clínico de San Carlos & 1 \\
\hline Hospital de la Santa Cruz y San Pablo & 1 \\
\hline Hospital de Sabadell & 1 \\
\hline Hospital del Mar & 1 \\
\hline Hospital General Universitario de Valencia & 1 \\
\hline Hospital Pío del Río Hortega & 1 \\
\hline Hospital Universitario Virgen del Rocío & 1 \\
\hline Hospital Virgen de la Arrixaca & 1 \\
\hline Universidad de Sevilla & 1 \\
\hline Provincia & Frecuencia \\
\hline Barcelona & 29 \\
\hline Madrid & 15 \\
\hline Gran Canaria & 6 \\
\hline Córdoba & 3 \\
\hline Valencia & 3 \\
\hline Alicante & 2 \\
\hline Sevilla & 2 \\
\hline Vizcaya & 2 \\
\hline Zaragoza & 2 \\
\hline Lérida & 1 \\
\hline Murcia & 1 \\
\hline Valladolid & 1 \\
\hline
\end{tabular}

en la asociación entre el tipo de documento y la provincia, al igual que no hemos encontrado diferencias al comparar el índice de cooperación, tipo de revista, idioma, y especialidad entre dos etapas de tiempo diferentes del periodo analizado (1992-96 vs 1997-2002).

\section{DISCUSIÓN}

Aunque la producción científica española en sistema respiratorio se ha incrementado claramente en los últimos años
TABLA IV

DISTRIBUCIÓN DE LOS DOCUMENTOS SEGÚN EL PROCEDIMIENTO DIAGNÓSTICO TERAPÉUTICO EN SISTEMA RESPIRATORIO

\begin{tabular}{lccc}
\hline Área temática & Frecuencia & $\begin{array}{c}\% \text { de } \\
\text { artículos }\end{array}$ & $\begin{array}{c}\text { \% área } \\
\text { temática }\end{array}$ \\
\hline FB-LBA & 29 & 43,3 & 30,5 \\
FB-catéter telescopado & 17 & 25,4 & 17,9 \\
Sólo FB & 13 & 19,4 & 13,7 \\
PAAF & 7 & 10,4 & 7,4 \\
FB con biopsia bronquial & 7 & 10,4 & 7,4 \\
Toracotomía & 6 & 9 & 6,3 \\
Toracoscopia-videotoracoscopia & 4 & 6 & 4,2 \\
FB con BTB & 4 & 6 & 4,2 \\
Trasplante pulmonar & 3 & 4,5 & 3,2 \\
FB con punción transtraqueal & 2 & 3 & 2,1 \\
FB con cepillado bronquial & 1 & 1,5 & 1,1 \\
FB con Laser YND & 1 & 1,5 & 1,1 \\
FB con punción transbronquial & 1 & 1,5 & 1,1 \\
\hline Total & 95 & & \\
\hline
\end{tabular}

FB-LBA: fibrobroncoscopia-lavado broncoalveolar; FB: fibrobroncoscopia; PAAF: punción aspirativa con aguja fina; BTB: biopsia transbronquial; YND: yag neodinio

(6-11), no observamos un aumento, a lo largo del perIodo estudiado, de la productividad en técnicas diagnóstico-terapéuticas relacionadas con dicho sistema. Recientemente se ha comunicado (9), al analizar la producción científica española en biomedicina y ciencias de la salud desde el año 1994 al 2000, que entre las disciplinas que incluye la medicina clínica destacan, con más de 1.000 documentos citables (por número de documentos), la hematología y la oncología seguidas de enfermedades infecciosas, sistema respiratorio, neurología clínica y, gastroenterología (que incluye hepatología), subrayando seguidamente la investigación en Salud Pública medioambiental y laboral. La evolución del sistema respiratorio se ha incrementado de una manera considerable, tanto en producción como en repercusión, pasando de un puesto intermedio en la producción total en la Medicina Clínica $(7,8)$, a encontrarse en la actualidad entre los más productivos $(9,10)$. Por el contrario, el cociente entre el número de citas recibidas y el número total de documentos citables $(\mathrm{C} / \mathrm{D})$ es más bajo en sistema respiratorio, pero en cambio, lo relacionado de sistema respiratorio con el área de urgencias y medicina intensiva, aunque con una menor producción, presenta un C/D elevado y con baja no citación de documentos. Mucho de lo encontrado en nuestro trabajo se ajustaría a este patrón, pues claramente el impacto relativo del área de técnicas respiratorias es mayor, aunque no hemos analizado la repercusión.

Otro aspecto a resaltar es el hecho de que la mayoría de los artículos que hemos localizado están publicados en inglés, y son preferentemente documentos originales y cartas al director. Como es conocido el idioma de publicación es un determinante de la probabilidad de recibir citas, un factor que se suma al determinado por el tipo de documento. Estos datos confirman la importancia actual del inglés como idioma primordial en la comunicación científica actual, por lo menos en cuanto al fenómeno de recibir citas (9). 
Igualmente, podemos comprobar, que aunque ampliamente distribuida por toda la geografía española, existe un predominio de la producción, en el campo analizado, en la Comunidad catalana, particularmente en el Hospital Clinic y Provincial de Barcelona, datos que no son diferentes a los obtenidos por otros investigadores (9).
Podemos concluir, que la producción en el área de técnicas diagnósticas y terapéuticas del sistema respiratorio se mantiene estable, sin una tendencia definida, a lo largo de los doce años analizados, lo que contrasta con el aumento de producción encontrado en el sistema respiratorio en general.

\section{Bibliografía}

1. García Río F. Estrategia para la búsqueda bibliográfica eficiente. Bibliometría. valoración crítica. Arch Bronconeumol 1999; 35 (Supl. 1): 27-30.

2. García Díaz F. Búsqueda de bibliografía médica a través de Internet. El proyecto PUB-MED. Med Clin (Barc) 1999; 113: 58-62.

3. Granda Orive JI, García Río F, Chillón Martín MJ, Escobar Sacristán J, Jareño Esteban J, Gutiérrez Jiménez T, et al. ¿Utilizamos adecuadamente las palabras clave en los originales? Análisis y evolución en tres áreas temáticas a través de Archivos de Bronconeumología (1994-2000) y concordancia con el Index Medicus. Arch Bronconeumol 2003; 39 (Supl. 2): 146.

4. Granda Orive JI, García Río F, Gutiérrez Jiménez T, Escobar Sacristán JA, Riera Palmero J, Callol Sánchez L. Evolution of Bibliometric Indicators and his websites evaluation approaches in relation to the foremost Respiratory Journal in Spanish. Cybermetrics 2004; 8 (1): paper1.<http://www.cindoc.csic.es/cybermetrics/articles/v8i1p1.html>.

5. Granda Orive JI, García Río F, Callol Sánchez L. Importancia de las palabras clave en las búsquedas bibliográficas. Rev Esp Salud Pública 2003; 77: 765-67.

6. Granda Orive JI, García Río F, Escobar Sacristán JA, Álvarez-Sala Walther R, Martínez Albiach JM, Callol Sánchez L. Evolución de la producción científica madrileña en revistas internacionales de sistema respiratorio de 1987 a 2001. Rev Patol Respir 2003; 6: 6-12.

7. Camí J, Fernández MT, Gómez Caridad I. La producción científica española en medicina y salud. Un estudio a través del Science Citation Index (1986-1989). Med Clin (Barc) 1993; 101: 721-31.

8. Camí J, Zulueta MA, Fernández MT, Bordons M, Gómez I. Producción científica española en biomedicina y ciencias de la salud durante el periodo 1990-93 (SCI y SSCI) y comparación con el periodo 1986-89. Med Clin (Barc) 1997; 109: 481-96.

9. Camí J, Suñen E, Carbó JM, Coma L. Producción científica española en biomedicina y ciencias de la salud (1994-2000). Informe del Instituto de Salud Carlos III-Fondo de Investigación Sanitaria. http://www.isciii.es/fis/mapa/index.htm

10. García Río F, Serrano S, Dorgham A, Álvarez-Sala R, Ruiz Peña A, Pino JM, et al. A bibliometric evaluation of European union research of the respiratory system from 1987-1998. Eur Respir J 2001; 17: 1175-80.

11. García Río F, Álvarez-Sala R, Gómez Mendieta MA, Ruiz Peña A, Serrano Peña S, Pino García JM, et al. Evolución de la producción científica española en revistas internacionales de sistema respiratorio de 1987-1998. Med Clin (Barc) 2000; 115: 287-293. 